\title{
Excitonics heats up
}

\author{
Until now, excitonic devices have only been realized at temperatures of $1.5 \mathrm{~K}$. Nature Photonics spoke to \\ Leonid Butov from the University of California in San Diego about his group's recent demonstration of \\ excitonic switches operating at $125 \mathrm{~K}$.
}

\section{What is an exciton?}

An exciton is a quasi-particle, typically created in a semiconductor, that consists of an electron-hole pair bound by the Coulomb force. The particularly useful feature of an exciton is that it can be generated by and converted back into a photon, opening the door for all sorts of ultra-small optical switches and modulators. We have been studying so-called indirect excitons. For a direct exciton in a quantum well (QW), both an electron and a hole are confined in the same QW. For an indirect exciton, however, the electron is confined in one QW and the hole is confined in another. The separation between these QWs is typically a few nanometres. Indirect excitons are particularly interesting because they can be electronically controlled like electrons in electronic devices, but can be easily and rapidly converted into photons. These properties pave the way for the development of new optoelectronic devices.

\section{Why are they interesting?}

We previously demonstrated that indirect excitons can be used in circuits in place of electrons. The potential advantages of excitonic devices include high operation and interconnection speed, small dimensions and the opportunity to increase the density of components in optoelectronic integrated circuits. The smallest dimensions achieved for conventional optical Mach-Zehnder modulators are $\sim 100 \mu \mathrm{m}$. In comparison, excitonic transistors have an architecture and operation principle similar to electronic field-effect transistors and have the potential to be similarly compact. In the excitonic transistor described in this issue, the distance between the source and drain was $3 \mu \mathrm{m}$, limited by the resolution of the lithography used for the sample processing. It is expected that the sizes of excitonic transistors can be reduced below $1 \mu \mathrm{m}$ - considerably smaller than the sizes of conventional photonic devices - by using electron beam lithography. The combined switching time and interconnection time for the first proof-of-principle excitonic transistor

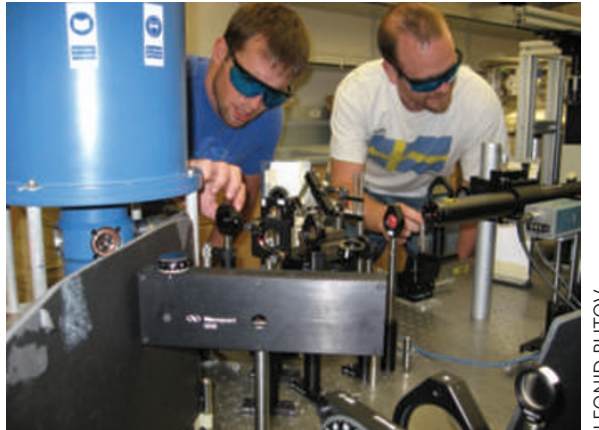

energy. In AlAs/GaAs, with only $3 \mathrm{~nm}$ between the QW centres, indirect excitons are characterized by high binding energies. This increased binding enables operation of excitonic devices, including modulation and on/off switching, at temperatures around $100 \mathrm{~K}$. This is an increase of two orders of magnitude in the operation temperature of excitonic devices, compared with the previous record. The operation principle of an exciton optoelectronic transistor is similar to that of an electronic fieldeffect transistor; it is a three-terminal

Alex High and Aaron Hammack from Leonid Butov's group at the University of California in San Diego adjust optics in one of their exciton experiments.

was $\sim 0.2 \mathrm{~ns}$, close to that achieved by ultrafast photonic modulators.

Excitonic devices potentially bring great improvements to the speed of electronic-optical interfaces. To transform an electronic signal into an optical one, an electron must be converted into a photon. This can be done by bringing a hole and electron together so that they recombine to produce a photon. In a conventional optoelectronic devices this takes time, causing an unwanted delay between signal processing in the electronics domain and communication in the optical domain. In an excitonic device, however, excitons can be converted into photons very rapidly and conveniently.

\section{Tell us about your recent success.} In the current work we moved to AlAs/ GaAs coupled-quantum-wells, compared with previous work where GaAs/AlGaAs was used. Exciton binding energy is of the order of $10 \mathrm{meV}$ and exciton lifetime is about $1 \mu$ s at low temperatures with zero applied voltage. The lifetime can be controlled by the applied voltage over several orders of magnitude. The problem with excitonic devices is that the finite exciton binding energy limits the operation temperature because excitons break into unbound electrons and holes at high temperatures, when thermal energy becomes comparable to the exciton binding device in which the exciton flux between two electrodes is controlled by a voltage applied to the third electrode. The inputs and outputs of excitonic devices may be photonic, where photons are converted to excitons at the input and excitons transform into photons at the output, or excitonic, where excitons arrive at the input from another excitonic device.

\section{What are the remaining challenges} and your future plans?

Our goal is to create efficient excitonic devices that are operational at room temperature. In particular, this requires the development of excitonic devices in structures where excitons have even longer lifetimes and higher binding energies than those currently attained. If all these challenges are resolved, excitonic devices may ultimately replace electronic devices in some applications. As the delay between signal processing and optical communication is effectively eliminated in excitonic devices, one can envisage excitonic devices replacing electronic devices in applications where a high interconnection speed is important. Excitonic signal processing and multiplexing may be examples, but we believe it is premature to discuss specific applications at this stage. The proof-of-principle for an excitonic transistor was demonstrated just recently and excitonics is still in an early stage of development.

\section{INTERVIEW BY DAVID PILE}

Leonid Butov and his colleagues have a Letter on excitonic switches on page 577 of this issue. 